\title{
MEDICAL APPLICATIONS OF ARTIFICIAL INTELLIGENCE
}

\section{AUTHOR - Dr. Rupanjan Roy}

POST GRADUATE TRAINEE, DEPARTMENT OF PERIODONTOLOGY, MANIPAL COLLEGE OF DENTAL SCIENCES, MANIPAL ACADEMY OF HIGHER EDUCATION, MANIPAL 


\begin{abstract}
The medical \& the dental field is a never-ending field of innovations \& developments and each time the researchers come up with something new. One such new dimension in the fields of medicine being the incorporation of Artificial intelligence assisted technologies improving diagnosis, treatment plan and treatment strategies. This review focusses on the application of different technologies of AI in different fields of medicine.
\end{abstract}

Keywords: ARTIFICIAL INTELLIGENCE, MEDICINE, APPLICATIONS

\title{
MEDICAL APPLICATIONS OF AI
}

\section{A. INTRODUCTION}

The concepts, techniques and tools of AI have been used in medical applications for over decades. The main aim of incorporating the technologies of AI was to improve the efficacy, consistency and the productivity. There is a gradual shift in consistent use of AI for tackling problems in medicine, mainly due to the improvement in accuracy and efficacy of AI techniques. One of the most important reasons in adopting AI in the medical field is primarily due to the availability of AI software.

AI techniques have been successful in the medical field and the technologies involving AI have been incorporated into the healthcare industry. The applications being wide \& diverse. Apart from the traditional applications of AI in the fields of medicine for diagnosis, patient data management, automatic classification, visualization etc., recently AI finds shows much more applications in the medical field. These include disease genes, wearable computing, hospital scheduling, medical robotics, surgery stimulation, artificial consciousness and much more.

The applications of AI in medicine are so widespread that there have literature surveys that have been conducted in order to study the AI applications in medical field. A study by Ramesh et al, 2004 (1) concluded that neural networks were the most widely used analytical tool. Another study by Yardimci in 2007 (2) concluded that fuzzy logic neural network is the most widely used analytical tool. In addition to all these, the more detailed and specific publications on AI can be broadly divided into two classes. The first set focusses on a specific set in AI, it being applied to different sets of medicine. However, the second set focusses more on the explicit areas of medicine and covers the applications of AI techniques in that area. Some examples of books in the first division include those with focus on fuzzy logic (Barro and Marin,2010). On the other 
hand, examples of book on the second set include medical informatics (Kelemen et al. 2008 \& Yoshida et al. 2010). (2)

\section{B. HISTORY}

Many early researches focused on the applications of AI in the medical field. Medical data collection and the use of digital data were discovered in the work of Ax (1960). (3) Holtzmann in 1960 further investigated and questioned the computing ability of the type of activities that supplement the clinical psychologists. Ledley \& Lusted, 1960 suggested that computers can be used as an adjunct in patient diagnosis, data recording and diagnostic testing. (4)

Medical applications of AI dates back to 1960s when one of the first published works in the field of medical applications of AI which had "artificial intelligence" in the title. The credit for this goes to Hunt, 1968 who for the very first time addressed the deductive and inductive problemsolving capabilities of AI in the field of psychology. Schwartz in his works in 1970 included the discussion on the impact of the "intellectual" use of computer in the fields of healthcare. Some of the other classical works of medical applications of AI include works done by Nordyke et al. 1971 who came up with an automated diagnosis of thyroid dysfunction. Shortliffe et al. In 1973 came up with the therapy of interactive advice giving with physicians. (5)

With these measurable improvements, the Medical artificial intelligence (MAI) shows great potential and promises of applying these AI technologies to analytical medical informatics, focused on the following areas:

* AI techniques in medicine

* Data Mining and knowledge discovery in medicine

* Medical expert systems

* Machine learning based medical systems

* Medical Signals and Image Processing Techniques

\section{APPLICATIONS (6)}

Alvira- Garcia et al. demonstrated the use of a neural network based multiscale Gaussian matching filter for the identification and segmentation on the images obtained from coronary angiogram \& X-ray and ultimately effectively enhanced the results of the image classification. 
$\square$ Cui et al. in his studies proposed a cascade neural network comprising of a Tumor localization network to localize brain tumors from slices from the slices of MRI images and also led to the invention of an Intra-Tumor classification network to label tumor regions. Slowly, with the advancement in technologies used to obtain, train and optimize these cascade neural networks, there is a better accuracy and computing efficacy of these networks over other neural networks.

Fu et al. published their results of a study where they have used a Convolutional Neural Network (CNN) for the recognition of a strabismus. The data was used to train the CNN, collected by an eye-movement tracker, including Gaze Deviation (GaDe) image data including both subjects of normal and strabismic visions. After the training with a large number of GaDe images, the CNN performance was successfully tested for strabismus recognition.

Chan et al. made use of a support vector machine in-order to quickly diagnose common pneumothorax by using the local binary patterns that have been obtained through a multiscale intensity texture analysis on the chest X-ray images.

Chen et al. designed a clinical decision support system to predict fractures in hip bones and vertebrates caused by medications for treatments of chronic respiratory diseases. An integrated genetic algorithm was incorporated within the system and support vector machine through training with balanced datasets obtained from random and cluster-based under sampling methods. The same was even tested with imbalanced datasets.

Barnes et al. led to the proposal of a data-driven predictive modeling framework for decision support systems, which is based on evolutionary computation techniques inorder to optimize multilevel data. An open-source software can be used to build the framework and it is made flexible to include other evolutionary algorithms.

Kim et al. proposed a Deep Belief Network and Dempster-Shafer- (DBN-DS-) based metaclassifier which is to be used for the pathologic prediction of prostate cancer, tested with data from thousands of patients. The results showed high accuracy $(81.27 \%)$ against the Partin table (64.14\%).

Kaur et al. did a thorough and detailed scrutiny of the hybrid computing models used to predict blood pressures (BP). The main constituent of each hybrid model was principal component analysis (PCA) and one of the following: Forward Stepwise Regression 
(FSWR), Artificial Neural Network (ANN), Adaptive Neuro-Fuzzy Inference System (ANFIS), and Least Square-Support Vector Machine (LS-SVM). The predicted BP was associated with reactivity to crossed legs among normotensive and hypertensive participants. \%e LS-SVM model achieved significant improvements.

Yang has done many studies with 3D Shape-Weighted Level Set Method (3D-SLSM) to carry out precise segmentation of tumors from 3D medical images. Errors and noise, largely affected the results of segmentation of most 3D segmentation algorithms; however, 3D-SLSM added 3D shape weighted values in each iterative process according to the volume changes. \%e accuracy of 3D-SLSM was tested with MRI and computergenerated images and resulted in showing the highest accuracy and lowest false-positive rate when compared with the standard tumor model.

Lin et al. researched on effects of outlier removals in medical datasets, that is, whether performing instance selection to filter out noisy data from a given training medical dataset has a positive impact on the final imputation results. By comparing the classification performance obtained through the processes combining instance selection and imputation and the baseline imputation process, three types of medical datasets are used: categorical, numerical, and mixed types. \%ree different instance selection methods and three model-based imputation algorithms were compared. \%e experimental results showed that performing instance selection first mostly improves the imputation result over the types of medical data. Further, the negative impact was to consider instance selection before imputation when the dataset contains lower dimensionalities and numbers of classes. However, for numerical datasets, the combined instance selection and imputation process would perform better than the baseline imputation process for most datasets with different missing rates. They also concluded that for mixed datasets, the instance selection effect was between that for categorical and numerical datasets, which meant that combining instance selection and imputation could be a better choice when algorithms were carefully chosen.

Chen devised a personalized antidiabetic medication recommendation system that tailored HbA1c levels to investigate medical differences for the selection of antidiabetic medications. The combination of fuzzy logic and ontology reasoning, could lead to the system producing a "patient-centric diabetes therapy" apart from acting as an adjunct to clinical diagnosis and assisting family practitioners in prescribing medications.

Alireza et al conducted a study in order to analyze the results of a machine learning based software as an effective platform for predicting the outcome after percutaneous nephrolithotomy (PCNL). The effectiveness of this system was compared with Guy's 
Stone Score (GSS) \& Clinical Research Office of Endourological Society (CROES) nomogram. In recent era of minimally invasive surgery, PCNL remains the gold standard for the management of large kidney stones. In order to analyze the stone free rate (SFR) post PCNL, it was Thomas and co-workers who first led to the invention of a GSS scale. This scale is a simple and a very basic qualitative measurement scale (Grade I-IV). The gradations are based on the shape, size, location of the stones as well as also by the presence or absence of renal or skeletal anomalies. This scale was further evolved \& modified by Smith \& co-workers who designed the CROES nomogram. In this type, several characteristics of the stone including size, number, burden and institute level volumes are included. An important feature of this CROES system being, it takes into consideration the weighting of all these parameters. This study was the first of it's kind to compare the outcome post PCNL using an artificial intelligent system with that of the available conventional approaches.

The results of the study showed that the machine learning system predicted the PCNL outcomes with an accuracy ranging between $80 \%$ and $95.1 \%$. For predicting the stonefree status, the AUC (Area under the curve) for the software (0.915) was significantly larger than the AUC for GSS (0.615) or CROES nomograms $(0.621)(\mathrm{p}<0.001)$. (7)

$\square$ Zhidong zhao et al conducted a study in-order to analyze the efficacy of a computer aided diagnosis system based on AI technology for monitoring Fetal Heart rate (FHR). They presented an 8-layer deep convolutional neural network (CNN) framework to automatically predict fetal acidemia. After signal preprocessing, the input 2-dimensional (2D) images were obtained using the continuous wavelet transform (CWT), which provided a better way to observe and capture the hidden characteristic information of the FHR signals in both the time and frequency domains. Unlike the conventional machine learning (ML) approaches, this work does not require the execution of complex feature engineering, i.e., feature extraction and selection. In fact, 2D CNN model can self-learn useful features from the input data with the prerequisite of not losing informative features, representing the tremendous advantage of deep learning (DL) over ML. So, once the training of the $\mathrm{CNN}$ model will be complete, the corresponding CAD tool can be used as an effective, accurate tool in order to predict fetal asphyxia objectively.

Medical 3D Bioprinting using AI technologies have shown a paradigm shift over the last few decades in terms of both in diversity in medical application as well as in the means of adopting it in daily investigations. The Radiological Society of North America Special Interest Group on 3D Printing (SIG) laid down few recommendations.

Consensus Methodology Recommendations: 
- 1-3 - red, rarely appropriate: There is a lack of a clear benefit or experience that shows an advantage over usual practice.

* 4-6 - yellow, maybe appropriate: There may be times when there is an advantage, but the data is lacking, or the benefits have not been fully defined.

* 7-9 - green, usually appropriate: Data and experience shows an advantage to 3D printing as a method to represent and/or extend the value of data contained in the medical imaging examination. (9)

$\square$ Prediction of Thromboembolic syndrome - Mahdieh et al conducted a study in order to method for improving the power of recognition and classification of thromboembolic syndrome based on the analysis of gene expression data using artificial neural networks. The results showed that when using auto-encoder networks, the classification accuracy was 93.12. When using the Principal component analysis (PCA) method to reduce the size of the data, the obtained accuracy was 78.26, and hence a significant difference in the accuracy of classification was observed. If auto-encoder network method is used, the sensitivity and specificity will be 92.58 and 93.68 and when PCA method is used, they will be 0.77 and 0.78 respectively. The results suggested that auto-encoder networks, compared with the PCA method, had a higher level of accuracy for the classification of thromboembolic syndrome status. (10)

Juan zhu et al conducted a systematic review in-order to analyze the effect of Distal acupoint stimulation based on AI technology \& peri-incisional stimulation for postoperative pain reduction after open abdominal surgery.

Flowchart Diagram: 


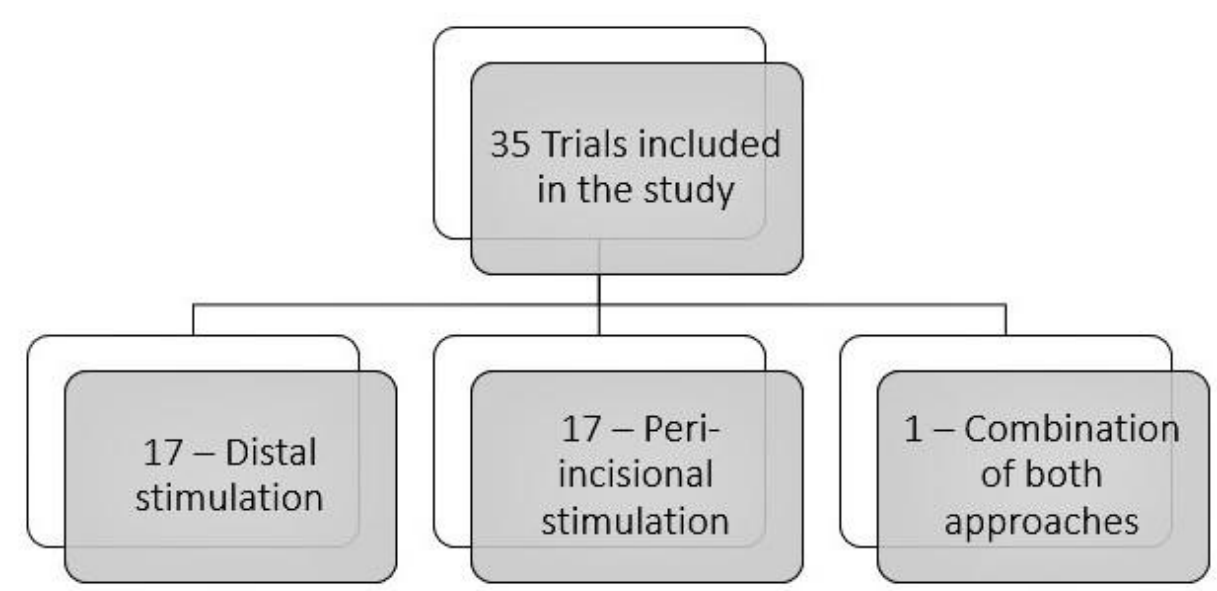

Subgroup analysis showed that both distal and peri-incisional stimulation significantly alleviated postoperative resting and movement pain from $4 \mathrm{~h}$ to $48 \mathrm{~h}$ after surgery by 6 to $25 \mathrm{~mm}$ on a $100 \mathrm{~mm}$ visual analogue scale
Peri-incisional stimulation showed a better reduction in postoperative opioid consumption

Fig 1: Table representing the study diagram in the systematic review and the group analysis.

The results of the systematic review showed that both distal and peri-incisional modes of stimulation were effective in reducing postoperative pain. (11)

$\square$ Optical Coherence Tomography (OCT) based deep learning algorithm for evaluation of treatment indications with anti-vascular endothelial growth factor medications - OCT based scans of the corneal retina helps in showcasing the detailed anatomical data and is a reliable tool for the doctors to come to a decision regarding antiVEGF indication. In the current years, holding the hands of AI technology, a deep convolutional artificial network is trained in-order to predict the treatment indication by analyzing the central corneal OCT scans without the need for a human intervention. 


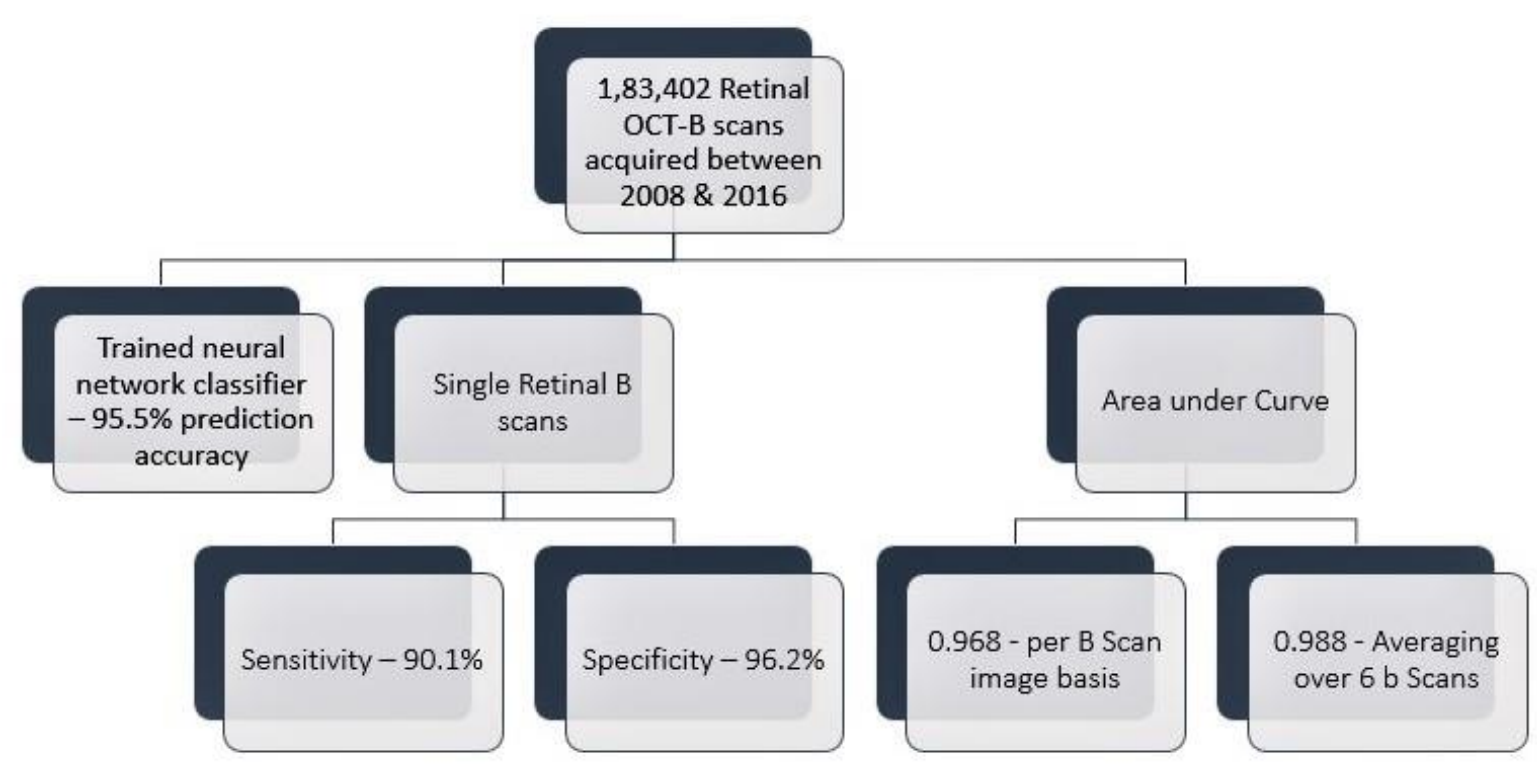

Fig 2: Table representing the study diagram and the group analysis.

The results of the study showed that deep artificial networks offer a paradigm shift on the classification of retinal OCT scans and supports the doctors in decision making process.

Medical Endoscopes - The technologies incorporating the use of AI finds massive application in the fields of medical endoscopy, ophthalmoscopy \& electron coloscopy. An AI application was created that delivered a signal in the forms of screen alert, location box etc. to an endoscope monitor which alerted the endoscopist to the area of suspected disease or most probable diagnosis. It also had the capacity to biopsy sample or resect the area endoscopically. (13)

RADIOLOGY - The use of CAD (computer-assisted diagnosis) in a screening mammography is well known. Recent studies have indicated that CAD is not of a lot of diagnostic aid, based on positive predictive values, sensitivity and speciality. In addition, the false-positive diagnoses may distract the radiologist resulting in unnecessary work-ups. (14)

DURGERIES - The Da Vinci robotic surgical system developed by Intuitive surgical has revolutionized the field of surgery especially urological and gynecological surgeries. The robotic arms of the system mimic a surgeon's hand movements with better precision and has a $3 \mathrm{D}$ view and magnification options which allow the surgeon to perform minute incisions. Since 2018, Buoy Health and the 
Boston children's hospital are collaboratively working on a web interface-based AI system that provides advice to parents for their ill child by answering questions about medications and whether symptoms require a doctor visit. The National Institute of Health (NIH) has created an AiCure App, which monitors the use of medications by the patient via smartphone webcam access and hence reduce nonadherence rates. (14)

\section{ADVANTAGES \& DISADVANTAGES OF AI IN MEDICAL FIELD}

The application of AI is definitely a problem-solving boon in the fields of medicine. Some of the notable advantages of AI in the field of medicine include thorough efficacy, accuracy, precision. Incorporation of AI technologies in the field of medicine has reduced the work-load which automatically made doctors give more time $\&$ attention on critical $\&$ important cases. Also, the advent of AI saves time, money \& helps in better patient monitoring.

However, with every advantage comes some disadvantages. Some of the disadvantages with the use of AI assisted technologies being it leads to the loss of jobs, lack of human empathy, lack of emotional intelligence.

Still with these disadvantages, AI with its humongous advantages paves the path to a new dimension in the fields of Medicine. (14) 


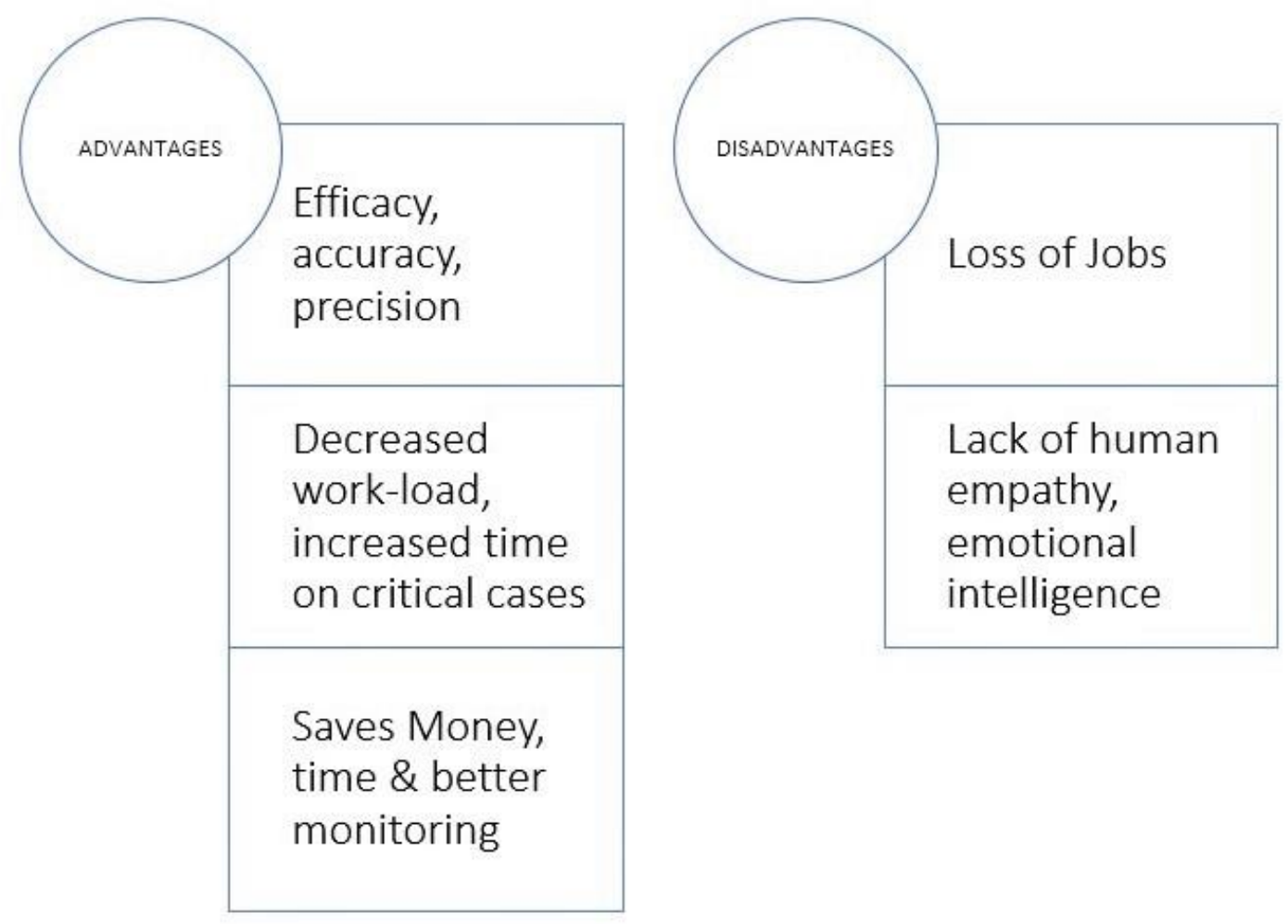

Fig 3: Flowchart depicting the advantages \& disadvantages of Al in medicine

\section{E. CONCLUSION}

The Medical \& the dental field is a field of innovations and developments. Holding the hands of science each time we come up with something new. One such new dimension is the application of AI in the field of medicine, which are vast and diverse. Almost, all branches of medicine are reliable either directly or indirectly on the technologies involving AI. AI is definitely one of the top advanced recent technologies implemented in the field of medical science with minimal equipment, intelligent parameters, at-par software and is revolutionizing and creating benchmarks in the field of medical diagnosis, treatment plan and treatment strategies.

\section{F. REFERENCES}


1. Holzinger A, Langs G, Denk H, Zatloukal K, Müller H. Causability and explainability of artificial intelligence in medicine. Wiley Interdisciplinary Reviews: Data Mining and Knowledge Discovery. 2019 Jul;9(4):e1312.

2. Hamet P, Tremblay J. Artificial intelligence in medicine. Metabolism. 2017 Apr 1;69:S36-40.

3. Szolovits P, editor. Artificial intelligence in medicine. Routledge; 2019 Mar 13.

4. Schwartz WB, Patil RS, Szolovits P. Artificial intelligence in medicine.

5. Briganti G, Le Moine O. Artificial intelligence in medicine: today and tomorrow. Frontiers in medicine. 2020 Feb 5;7:27.

6. Yung-Kuan Chan, Yung-Fu Chen, Tuan Pham, Weide Chang, Ming-Yuan Hsieh, "Artificial Intelligence in Medical Applications", Journal of Healthcare Engineering, vol. 2018, Article ID 4827875, 2 pages, 2018

7. Alireza Aminsharifi, Dariush Irani, Sona Tayebi, Taher Jafari Kafash, Tayebeh Shabanian, and Hossein Parsaei.Journal of Endourology.Jun 2020.692-699.

8. Zhao Z, Deng Y, Zhang Y, Zhang Y, Zhang X, Shao L. DeepFHR: intelligent prediction of fetal Acidemia using fetal heart rate signals based on convolutional neural network. BMC Med Inform Decis Mak. 2019 Dec 30;19(1):286. doi: 10.1186/s12911-019-1007-5. PMID: 31888592; PMCID: PMC6937790.

9. Chepelev, L., Wake, N., Ryan, J. et al. Radiological Society of North America (RSNA) 3D printing Special Interest Group (SIG): guidelines for medical 3D printing and appropriateness for clinical scenarios. 3D Print Med 4, 11 (2018).

10. Khalili M, Alavi Majd H, Khodakarim S, Ahadi B, Hamidpour M. Prediction of the Thromboembolic Syndrome: an Application of Artificial Neural Networks in Gene 
Expression Data Analysis. Arch. Adv. Biosci. [Internet]. 2016Mar.12 [cited 2021Mar.2];7(2):15-2

11. Zhu J, Xu Q, Zou R, Wu W, Wang X, Wang Y, Ji F, Zheng Z, Zheng M. Distal acupoint stimulation versus peri-incisional stimulation for postoperative pain in open abdominal surgery: a systematic review and implications for clinical practice. BMC Complement Altern Med. 2019 Jul 30;19(1):192. doi: 10.1186/s12906-019-2583-8. PMID: 31362730; PMCID: PMC6668193.

12. Prahs P, Radeck V, Mayer C, Cvetkov Y, Cvetkova N, Helbig H, Märker D. OCT-based deep learning algorithm for the evaluation of treatment indication with anti-vascular endothelial growth factor medications. Graefes Arch Clin Exp Ophthalmol. 2018 Jan;256(1):91-98. doi: 10.1007/s00417-017-3839-y. Epub 2017 Nov 10. PMID: 29127485.

13. Agah A, editor. Medical applications of artificial intelligence. CRC Press; 2013 Nov 6.

14. Amisha, Malik P, Pathania M, Rathaur VK.Overview of artifcial intelligence medicine. J Family Med Prim Care2019;8:2328-31 